\title{
Reflection properties of metallic photonic crystals
}

Article in Applied Physics A · March 1998

DOI: $10.1007 / \mathrm{s} 003390050679$

CITATIONS

17

6 authors, including:

\section{Ekmel Ozbay}

Bilkent University

634 PUBLICATIONS 15,227 CITATIONS

SEE PROFILE

\section{Gary Tuttle}

lowa State University

75 PUBLICATIONS 3,139 CITATIONS

SEE PROFILE

\section{READS}

33

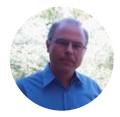

Michael M Sigalas

University of Patras

158 PUBLICATIONS 9,196 CITATIONS

SEE PROFILE

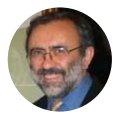

Costas M. Soukoulis

lowa State University

563 PUBLICATIONS 35,432 CITATIONS

SEE PROFILE

Some of the authors of this publication are also working on these related projects:

Project Electrodynamic Modeling of Quantum Dot Luminescence in Plasmonic Metamaterials View project

Project $\quad$ Loss compensation of plasmonic losses View project 


\title{
Rapid communication
}

\section{Reflection properties of metallic photonic crystals}

\author{
B. Temelkuran ${ }^{1}$, E. Ozbay ${ }^{1}$, M. Sigalas ${ }^{2}$, G. Tuttle ${ }^{2}$, C.M. Soukoulis ${ }^{2}$, K.M. Ho ${ }^{2}$ \\ ${ }^{1}$ Department of Physics, Bilkent University, Bilkent, Ankara 06533, Turkey \\ (Fax: +90-312/266-4579, E-mail: ozbay@fen.bilkent.edu.tr) \\ ${ }^{2}$ Ames Laboratory and Microelectronics Research Center, Iowa State University, Ames, IA 50011, USA \\ (Fax: +1-515/294-0665, E-mail: sigalas@iastate.edu)
}

Received: 10 November 1997/Accepted: 16 November 1997

\begin{abstract}
We measured reflection-magnitude and reflectionphase properties of metallic photonic crystals. The experimental results are in good agreement with the theoretical calculations. We converted the reflection-phase information to an effective penetration depth of the electromagnetic waves into the photonic crystal. This information was then used to predict resonance frequencies of defect structures. A symmetric resonant cavity was built, and an experimental set-up limited reflection magnitude of $80 \mathrm{~dB}$ below the incident signal was observed at resonance frequency.
\end{abstract}

PACS: 42.50.-p; 41.20.Jb; 71.25.Cx; 84.90.+a

Propagation of electromagnetic (EM) waves in periodic dielectric structures can be completely forbidden for a certain range of frequencies [1-3]. These three-dimensional arrays - photonic band gap (PBG) crystals - can be used to engineer the properties of the radiation field within these structures [4-13]. Although the earlier work on photonic crystals concentrated on building structures with dielectric materials, there are certain advantages of introducing metals to photonic crystals [14-19]. First, the metals offer a higher rejection rate per layer when compared to dielectric crystals. Second, for microwave applications the dimensions of metallic crystals can be kept much smaller than the minimum dimensions needed for a typical dielectric crystal. In this paper, we investigate the reflection properties of layer-by-layer metallic photonic crystals, and use these properties to predict defect formation in layer-by-layer metallic photonic crystals.

In our investigations of reflection properties, we used metallic photonic crystals with the simple-tetragonal (st) structure shown schematically in Fig. 1. This structure has a two-layer unit cell in the stacking direction. The metallic rods of the structure were $0.8 \mathrm{~mm}$ wide, $2.5 \mathrm{~mm}$ thick, and $120 \mathrm{~mm}$ long, with a center-to-center distance (between adjacent parallel rods) of $7.6 \mathrm{~mm}$. In our earlier work, we investigated the transmission properties of this metallic structure and measured a band gap with an upper edge at $20 \mathrm{GHz}$ and a lower edge extending down to zero frequencies. Within the band gap, the crystal exhibited a typical rejection rate of

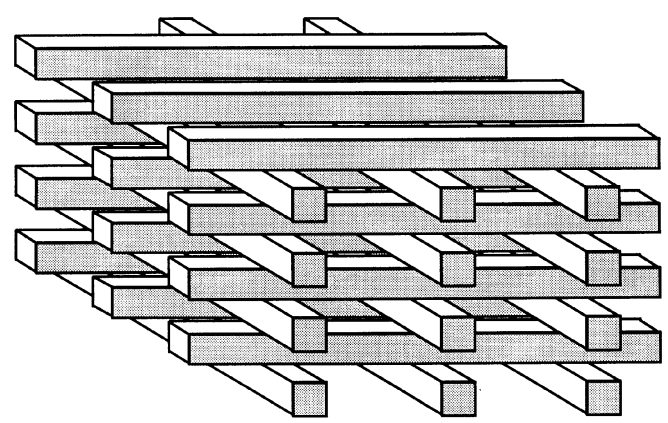

Fig. 1. Schematics of simple tetragonal layer-by-layer photonic band gap crystal

$7 \mathrm{~dB}$ per layer [20]. An HP 8510C network analyzer and three standard-gain microwave horn antennas were used for measurement of transmission and reflection properties [21]. The reflection calibration was performed by a metal sheet, which was assumed to be a perfect reflector at the measurement frequencies.

The reflection and transmission amplitude characteristics of a 6 layer crystal along the stacking direction with an incidence angle $\theta=5^{\circ}$, is shown in Fig. 2a. We also theoretically investigated the reflection properties of the metallic photonic crystals. The transfer-matrix method [22,23] (TMM) was used to calculate the EM transmission and reflection through the metallic structures. Figure 2a compares the theoretical reflection and transmission characteristics of the 6-layer-thick crystal with the experimental results. As can be seen from the plot, the theory and experiment were in good agreement. Although the reflection-magnitude properties of the crystal were independent of the polarization vector $\boldsymbol{e}$ of the incident EM wave, we found a strong polarization dependence for the phase of the reflected waves. Figure $2 b$ shows the phase of the reflected waves as a function of frequency for both polarizations, where the polarization vector $\boldsymbol{e}$ of the incident EM wave is either perpendicular or parallel to the rods of the top layer of the photonic crystal. The phase difference between two polarizations is close to $90^{\circ}$ throughout the photonic band gap frequencies. 

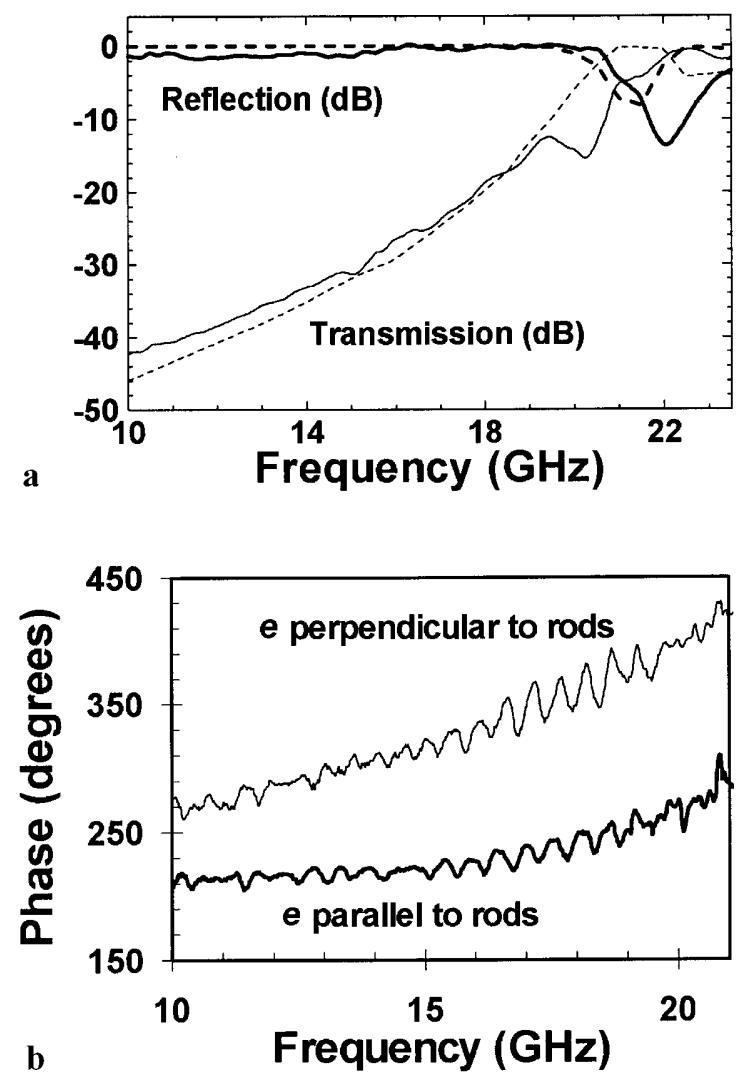

Fig. 2. a Comparison of the theoretical (dashed) and experimental (solid) reflection and transmission characteristics of the metallic photonic crystal. b Experimental reflection-phase properties of the photonic crystal for different polarizations

This phase information can also be interpreted as a penetration depth of the EM waves into the metallic photonic crystal. To account for the phase delay due to the reflection phase, the EM waves can be considered to penetrate a certain distance in the crystal and then reflect back from an ideal metallic plane with a phase shift of $180^{\circ}$. The distance from the surface to the reflection plane $L_{\text {eff }}$ can be formulated as

$L_{\mathrm{eff}}=\frac{c}{2 f}\left(\frac{\phi-180}{360^{\circ}}\right)$

where $f$ is the frequency, $\phi$ is the reflection phase (at frequency $f$ ) of the surface, and $c$ is the speed of light. This interpretation can be used to predict the defect frequencies of planar defect structures. Let us assume that two metallic photonic crystal surfaces with effective reflection plane distances of $L_{\text {eff1 }}$ and $L_{\text {eff2 }}$ are brought together to form a Fabry-Perot cavity with a separation width of $L_{\text {cav }}$. The cavity can be considered to have an effective total cavity length of $L_{\mathrm{tot}}=L_{\mathrm{eff} 1}+L_{\mathrm{eff} 2}+L_{\mathrm{cav}}$. So, the resonance is expected to occur, when half of a wavelength and its integer multiples are equal to $L_{\text {tot }}$. The resonance frequency $f_{\text {res }}$, called the defect frequency, can be written as

$$
f_{\text {res }}=\frac{m c}{2 L_{\mathrm{tot}}}=\frac{m c}{2}\left(\frac{1}{L_{\mathrm{cav}}+L_{\mathrm{eff} 1}+L_{\mathrm{eff} 2}}\right), \quad m=1,2,3 .
$$

We experimentally tested this argument by separating a 12-layer photonic crystal from the middle with a separation width of $L_{\text {cav }}$. We then measured the transmission properties and the corresponding defect frequency $f_{\text {res }}$. The knowledge of the defect frequency can be used to predict the sum of effective reflection plane distances $L_{\text {eff,tot }}$ by using the following relation:

$L_{\text {eff,tot }}=L_{\text {eff } 1}+L_{\text {eff } 2}=\frac{m c}{2 f_{\text {res }}}-L_{\text {cav }}, \quad m=1,2,3$.

We then measured the reflection-phase properties of the two 6-layer mirrors for both polarizations, and calculated $L_{\text {eff,tot }}$ by using the relations in (1) and (3). The values obtained from the defect frequency measurements (of cavities with different separation lengths) and the reflection phase measurements are compared in Fig. 3. As can be seen from the plot, there is good agreement between the predicted

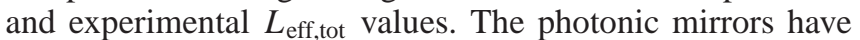
a $L_{\text {eff,tot }}$ typically around $5 \mathrm{~mm}$ within the band gap frequencies. This information can easily be used to design a defect structure with a given frequency. As an example, a cavity with a resonance at $15 \mathrm{GHz}$ should have an effective total cavity length $\left(L_{\text {tot }}\right)$ of $10 \mathrm{~mm}$ (corresponding to $m=1$ ). At this frequency $L_{\text {eff,tot }}=5 \mathrm{~mm}$, and according to (3), the cavity separation width $L_{\text {cav }}$ should be chosen as $5 \mathrm{~mm}$. When we built a cavity with a separation length of $5 \mathrm{~mm}$, we measured a defect frequency at $14.85 \mathrm{GHz}$ which was very close to the design frequency. The same frequency can also be obtained by using the second resonance $(m=2)$ of a different cavity, where the new effective total cavity length is twice as much or $L_{\text {tot }}=20 \mathrm{~mm}$. So a cavity with $L_{\text {cav }}=15 \mathrm{~mm}$ should also yield a defect frequency near $15 \mathrm{GHz}$. When we built a cavity with this separation width, we measured a defect frequency at $14.95 \mathrm{GHz}$, which further confirmed the usefulness of our prediction technique.

We also measured the reflection properties of the planar defect structures. As the structures were obtained by separating a photonic crystal from the middle, they can be considered as Fabry-Perot resonators with symmetric mirrors. For a symmetric Fabry-Perot resonator (which is also called the matched case), one expects all of the incident power to be transmitted (which means zero reflection) at the resonance frequency [24]. In order to test this argument for planar defect

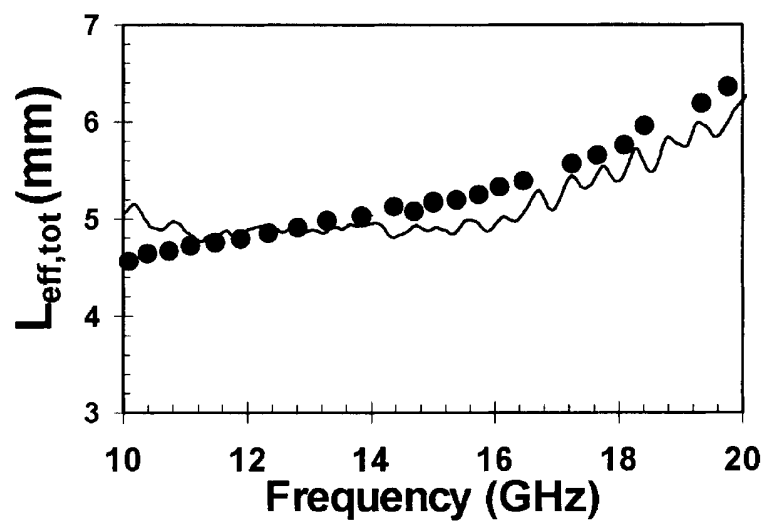

Fig. 3. Comparison of the experimental (circles) and predicted effective reflection plane distances (solid line) of the Fabry-Perot cavity 

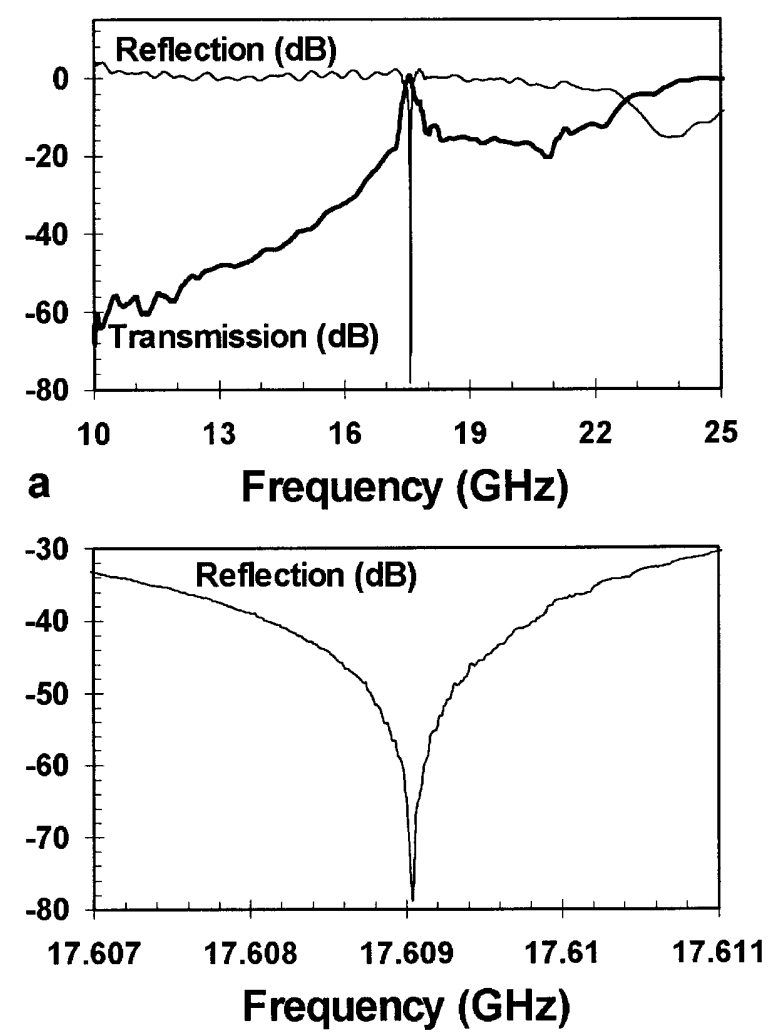

Fig. 4. a Reflection and transmission amplitude properties of a symmetric planar defect structure. b Reflection-amplitude properties of the structure with a zoomed frequency scale

structures, we built a cavity by separating an 8-layer crystal from the middle with a separation width of $2.5 \mathrm{~mm}$. The reflection and transmission properties of this planar defect structure is shown in Fig. 4. As can be seen from the plot, at resonance all of the incident power is transmitted and the reflection amplitude drops to an experimental set-up-limited value of $-80 \mathrm{~dB}$. This strong reflection property can be effectively used for reflection-type filtering applications.

In summary, we have theoretically and experimentally investigated the surface-reflection properties of metallic photonic crystals. We have converted the reflection-phase information into an effective penetration depth and used this depth to predict resonance frequencies of defect structures. The agreement between the prediction and the experiment is very good, confirming the validity of the Fabry-Perot cavity model used for the defect structures. To our knowledge, our measurements are the first-reported reflection-phase measurements of metallic photonic crystals in the scientific literature.

Acknowledgements. This work is supported by the Turkish Scientific and Technical Research Council (TÜBITAK) under contract No. EEEAG156, National Science Foundation Grant No. INT-9512812, and NATOCollaborative Research Grant No. 950079. Ames Laboratory is operated for the US Department of Energy by Iowa State University under contract No. W-7405-Eng-82.

\section{References}

1. E. Yablonovitch, T.J. Gmitter, K.M. Leung: Phys. Rev. Lett. 67, 2295 (1991)

2. K.M. Ho, C.T. Chan, C.M. Soukoulis: Phys. Rev. Lett. 65, 3152 (1990)

3. For a review, see the articles in Photonic Band Gap Materials, ed. by C.M. Soukoulis (Plenum, New York 1996)

4. U. Gruning, V. Lehmann, S. Ottow, K. Busch: Appl. Phys. Lett. 68, 747 (1996)

5. T.F. Krauss, R.M. De La Rue: Appl. Phys. Lett. 68, 1613 (1996)

6. E. Ozbay, J.S. McCalmont, G. Tuttle, R. Biswas, M.M. Sigalas, C. Soukoulis, K.M. Ho: Appl. Phys. Lett. 67, 1969 (1995)

7. S.-Y. Lin, V.M. Hietala, S.K. Lyo, A. Zavlavsky: Appl. Phys. Lett. 68, $3233(1996)$

8. S. Noda, N. Yamamoto, A. Sasaki: Jpn. J. Appl. Phys. 35, 909 (1996)

9. P.J. Roberts, T.A. Birks, P.St. Russell, T.J. Shepherd, D.M. Atkin: Opt. Lett. 21, 507 (1996)

10. E. Ozbay: J. Opt. Soc. Am. B 13, 1945 (1996)

11. M.C. Wanke, O. Lehmann, K. Mueller, Q. Wen, M. Stuke: Science 275, 1284 (1997)

12. R.J. Pradhan, I. Inanc Tarhan, G.H. Watson: Phys. Rev. B 54, 11245 (1996)

13. E. Ozbay, E. Michel, G. Tuttle, M. Sigalas, R. Biswas, K.M. Ho: Appl. Phys. Lett. 64, 2059 (1994)

14. E.R. Brown, O.B. McMahon: Appl. Phys. Lett. 67, 2138 (1995)

15. M. Sigalas, C.T. Chan, K.M. Ho, C.M. Soukoulis: Phys. Rev. B 52, 11744 (1995)

16. D.F. Sievenpiper, M.E. Sickmiller, E. Yablonovitch: Phys. Rev. Lett. 76, 2480 (1996)

17. S. Fan, P.R. Villenevue, J.D. Joannopoulos: Phys. Rev. B 54, 11245 (1996)

18. D.R. Smith, S. Shultz, N. Kroll, M. Sigalas, K.M. Ho, C.M. Soukoulis: Appl. Phys. Lett. 65, 645 (1994)

19. J.S. McCalmont, M. Sigalas, G. Tuttle, K.M. Ho, C.M. Soukoulis: Appl. Phys. Lett. 68, 2759 (1996)

20. E. Ozbay, B. Temelkuran, M. Sigalas, G. Tuttle, C.M. Soukoulis, K.M. Ho: Appl. Phys. Lett. 69, 3797 (1996)

21. E. Ozbay, B. Temelkuran: Appl. Phys. Lett. 69, 743 (1996)

22. J.B. Pendry, A. MacKinnon: Phys. Rev. Lett. 69, 2722 (1992)

23. M.M. Sigalas, C.M. Soukoulis, E.N. Economou, C.T. Chan, K.M. Ho: Phys. Rev. B 48, 14121 (1993)

24. A.E. Siegman: Lasers (University Science Books, Mill Valley 1986) 